ARTICULOS ORIGINALES

Rev Chil Salud Pública 2020,

Vol 24(1): 49-54

\section{DISTRIBUCIÓN ETARIA, ANTECEDENTES FAMILIARES Y ESTADIO DEL CÁNCER DE MAMA EN MUJERES ASISTIDAS EN HOSPITALES PÚBLICOS EN CÓRDOBA: 2004 - 2010.}

\author{
AGE DISTRIBUTION, FAMILY HISTORY, AND STAGE OF BREAST \\ CANCER IN WOMEN TREATED IN PUBLIC HOSPITALS IN CÓRDOBA, \\ ARGENTINA: 2004 - 2010.
}

\section{RESUMEN}

Introducción: El objetivo del presente estudio fue analizar la edad de la mujer al momento del diagnóstico de cáncer de mama, el estadio del mismo y los antecedentes familiares de cáncer de mama en las mujeres que consultaron en el ámbito público de la Ciudad de Córdoba durante el período 2004-2010.

Material y métodos: El presente fue un estudio observacional efectuado en cuatro instituciones públicas, a partir del total de datos de los Servicios de Anatomía Patológica y Estadística registrados en las historias clínicas ( $n=1423)$. Se evaluó la tendencia de edad de diagnóstico con regresión lineal y el tipo histológico y grado del tumor según la edad de la mujer, con regresión de Poisson. Resultados: En relación a la edad del diagnóstico, el 8,74\% de las mujeres estudiadas eran menores a 40 años, lo cual representa una mayor proporción a los valores informados en la literatura mundial. En relación a la distribución por edad del total de pacientes con cáncer de mama, la mayor frecuencia fue en el grupo de 50 a 59 años. Discusión: La proporción de mujeres mayores de 40 años detectadas en estadio tardío fue el $44,22 \%$, siendo superior a los valores informados en los países desarrollados. La tendencia de edad de diagnóstico se incrementó en un 0,39 por año, y no hubo cambio en el tipo del estadio. En términos de antecedentes familiares, se detectó la presencia en el 15,95\% de los casos, si bien es de destacar que la frecuencia de registro de estos antecedentes en las historias clínicas fue limitada.

Palabras claves: cáncer mamario; investigación sanitaria; grupo etario
Ana María Juárez

Ruth Fernández Escuela de Salud Pública, Facultad de Ciencias Médicas, Universidad

Nacional de Córdoba aruthfernandez@gmail.com

Recibido el 21-07-2019 Aceptado el 12-12-2019

\section{ABSTRACT}

Introduction: The aim of this study was to analyze the age at which women treated in public hospitals in the City of Córdoba, Argentina from 2004 to 2010 were diagnosed with breast cancer, their stage at diagnosis, and their family history of breast cancer.

Material and methods: This study was carried out in four public institutions, using the total number of cases registered in medical records by the pathology and statistical services $(n=1423)$. Trends in age at diagnosis over the study period was evaluated with linear regression, and tumor stage and histology according to age was analyzed with Poisson regression.

Results: In terms of diagnostic age, $8.74 \%$ of the women studied were under 40 years of age, which represents a higher percentage of cases, in comparison with international literature. With regards to age distribution, the greatest number of cases was observed in the group between 50 and 59 years of age.

Discussion: The proportion of women older than 40 years detected with late stage cancer was $44.22 \%$, which is higher than the values reported in developed countries. The diagnostic age trend increased by 0.39 per year, and there was no change in the stage at diagnosis. Finally, $15.95 \%$ of the women had reported family history of breast cancer, although it is noteworthy that the frequency of recording family history in the medical charts was limited.

Key word: breast neoplasms; health services research; age groups 


\section{INTRODUCCIÓN}

La epidemiología clínica y genética son imprescindibles para detectar patologías con diagnóstico precoz. El control del cáncer ha tenido éxito en los países de altos ingresos, con datos de alta calidad, en contraste con aquellos de medianos y bajos ingresos, donde los costos de atención sanitaria son insostenibles y los datos son de baja calidad1. La epidemiología en el extenso campo de la salud pública estudia la historia natural de la enfermedad, descubre los factores etiológicos, identifica los factores causales e indaga los aspectos ambientales ${ }^{1}$. El valor epidemiológico sobre la concientización en la población femenina con cáncer de mama tiene sus ventajas al incentivar el screening, disminuyendo la carga de enfermedad (definida como la medida de las pérdidas de salud) ${ }^{2}$ sometiéndose a controles anuales, que permite detectar la enfermedad en etapas más tempranas ${ }^{3}$. Para detectar tempranamente la patología lo más adecuado es la mamografía que identifica entre el $80 \%$ y el $90 \%$ de los cánceres de mama permitiendo la detección precoz y el tratamiento oportuno de la patología4-5. En los países del continente americano, la tasa de mortalidad por cáncer de mama oscila entre un 9,7 por 100.000 para Ecuador y 24,1 por 100.000 para Uruguay1. Para el año 2012 el cáncer de mama fue la primera causa de muerte en la mujer en Chile ${ }^{2,6}$. La epidemiología del cáncer de mama en la Argentina muestra una incidencia estimada de 7/100.000 mujeres ${ }^{1}$, siendo la segunda tasa de mortalidad por cáncer de mama en el continente $(21,8 / 100.000)^{8}$. El Registro de Tumores de la Provincia Córdoba (Argentina) refiere que el cáncer de mama resultó ser el más frecuente en las mujeres, durante el período 2004/20099.

La edad promedio de aparición del cáncer de mama muestra variaciones en los estudios internacionales. Así, el promedio de edad referido para las mujeres norteamericanas es de 60 años $^{10}$, en estudios cubanos 58 años $^{11}$, en estudios iraníes 46 años $^{12}$, en estudios chilenos 66 años ${ }^{13}$.

La patología aumenta con la edad, pero las mujeres de edad menor o igual a 39 años presentan la particularidad de tener una mayor carga de enfermedad y mortalidad, puesto que biológicamente la enfermedad es más agresiva y su diagnóstico se realiza en estadios más avanzados ${ }^{14,15}$. Definir el concepto de "mujer joven" no es uniforme en el campo de la patología maligna de mama; diferentes artículos hacen referencia desde los 35 a 40 años, hasta 45 años $^{16}$. La mayoría de los artículos consultados en mujeres menores de 40 años refieren una prevalencia de $5 \%$ a $7 \%$, siendo el tipo de cáncer más frecuentemente diagnosticado en mujeres de la franja de 25 a 39 años de edad ${ }^{17,18}$.

El cáncer en la mujer menor de 40 años es una entidad diferente del que se presenta en mujeres de franjas etarias superiores, por presentar ambas epidemiologías distintas. En los países desarrollados con políticas sanitarias efectivas, se les diagnostica la patología mamaria en estadios 0 y I en el $55 \%$ de las mujeres, el $35 \%$ en estadio II, el $6 \%$ en estadio III y el $5 \%$ en estadio IV. Por el contrario, en India el $74 \%$ de los tumores de mama diagnosticados están localmente avanzados o metastásicos. No se ha encontrado acuerdos en la literatura, ni una definición, que establezca los límites entre el cáncer de mama de diagnóstico temprano y el comienzo del diagnóstico tardío. Las referencias son contrapuestas, así el Instituto Nacional del Cáncer indica como diagnóstico temprano el "cáncer de mama que no se ha diseminado más allá de la mama o los ganglios linfáticos de la axila; incluye el carcinoma ductal in situ y los cánceres de mama en estadios I, IIA, IIB y III $A^{\prime \prime}$, en cambio en un estudio retrospectivo Hayat $\mathrm{M}$ J. y col. opinan que el factor pronóstico del cáncer de mama en estadio temprano es la falta de impacto de la enfermedad en los ganglios linfáticos axilares $^{19}$.

Con el advenimiento de las pruebas genéticas en la práctica oncológica se logró detectar la susceptibilidad de familias de alto riesgo. La predisposición hereditaria constituye un factor de riesgo no modificable; las mutaciones genéticas y sus historias familiares están presentes en el cáncer de mama en mujeres con antecedentes de cáncer en sus abuelas, tías o primas, y en familias con cánceres múltiples ${ }^{20}$.

Con la finalidad de identificar las características en la tendencia de los antecedentes de cáncer de mama registrados en hospitales públicos de la ciudad de Córdoba (Argentina), este estudio se propuso como objetivo analizar la edad de la mujer al momento del diagnóstico de cáncer de mama, el estadio del mismo y los antecedentes familiares de cáncer de mama en las mujeres que consultaron en al ámbito público durante el período 2004-2010.

\section{MATERIAL Y MÉTODO}

Descripción del ámbito de estudio. La fuente de datos para el análisis de la presente investigación fue- 
ron el total de historias clínicas ( $n=1493$ ) con informes anatomopatológicos de pacientes con diagnóstico de cáncer de mama en los archivos de los Servicios de Anatomía Patológica en la ciudad de Córdoba, de cuatro instituciones públicas de la ciudad (tres hospitales generales y una maternidad). Estas instituciones son las referentes de la patología mamaria del sistema público en la ciudad de Córdoba.

Instrumento de recolección de datos. De las fichas del Servicio de Anatomía Patológica de las instituciones en estudio se obtuvo información acerca de la edad de las mujeres al momento del diagnóstico de cáncer, el grado y diferenciación anatomopatológica del tumor, el estadio clínico del cáncer de mama, definido según clasificación TNM (E0, El, EII, EIII, EIV), y antecedentes familiares de $1^{\circ}$ y $2^{\circ}$ grado.

Análisis de los datos Las variables mensurables se describieron a partir de sus medidas de resumen, y las variables categóricas se describen a partir de análisis de frecuencias y sus comparaciones con la técnica de datos categorizados, utilizando el test de Chi-cuadrado ajustado. Para evaluar el nivel de detección temprana de los casos registrados durante el período 2004/2010 se analizaron los registros por año y se relacionó entre ellos la edad de diagnóstico de las mujeres incluidas en el estudio. Este procedimiento luego fue efectuado para cada institución. La edad de diagnóstico fue analizada por década.

Para evaluar las tendencias de edad de diagnóstico durante el período analizado se utilizó el modelo de regresión lineal, considerando la edad de la mujer como variable respuesta y el año de registro como variable regresora. Para evaluar la posibilidad de cambios de tendencia en el tipo histológico y grado del tumor registrado según la edad de la mujer, se utilizó el modelo de regresión de Poisson, donde la variable dependiente fue el año del registro, la variable regresora fue la edad y las variables clasificadoras, el tipo histológico y el grado de diferenciación tumoral.

La identificación de las características de antecedentes familiares de pacientes con cáncer de mama en el período 2004-2010 fue analizada mediante la presentación de la frecuencia de cada categoría asignada al tipo de antecedente de primero o segundo grado de parentesco.

En todos los casos se trabajó con un nivel de significación de 0,05 y todos los análisis fueron procesados utilizando el software estadístico InfoStat.

Consideraciones éticas: La presente investigación tuvo en cuenta los resguardos éticos relativos a la identidad de las mujeres registradas en los informes anatomopatológicos y en la historias clínicas. Se cumplió con la normativa nacional e internacional para la ética de trabajos basados en datos secundarios.

\section{RESULTADOS}

Se analizaron 1493 fichas clínicas las cuales correspondieron a los registros de las cuatro instituciones durante el período 2004-2010. De las mismas el $63,63 \%$ contenían datos completos acerca del estadio tumoral y sólo el $19 \%$ de las historias clínicas presentaban los antecedentes familiares.

La edad fue analizada por década y por año de estudio observándose que el diagnóstico de cáncer de mama en mujeres con una edad igual o menor a 39 años presentó una frecuencia de 8,74\% en el total de los hospitales generales incluidos en el estudio. La frecuencia de diagnósticos efectuados en las mujeres en la década de 30-39 años fue superior a la década de 20-29 años ( $p<0,0001)$. En la maternidad, la frecuencia de registros de las pacientes con igual o menor a 39 años fue de $11,24 \%$, lo cual supera a los registros en los hospitales generales analizados $(p<0,0001)$. En estos últimos, el predominio de grupo de edad de diagnóstico fue entre 50-59 años $(p<0,0001)$. En las siguientes décadas la frecuencia van disminuyendo $(p<0,0001)$.

En cuanto a la distribución de los registros de estadios tumorales en relación a la edad de diagnóstico de las 950 historias clínicas que tenían los datos completos (Tabla 1), se detectó que el diagnóstico en mujeres menores de 39 años fue de 86 casos (9,05\%). En el grupo de $20-29$ años el $70 \%$ fue diagnosticado en estadio EIIB, EIII, EIV; en el grupo de 30-39 años, el 42,11\% fue diagnosticado en E0, I, IIA y el $57,89 \%$ en EIIB, EIV. Del total de mujeres con diagnóstico efectuado a partir de los 40 años $(90,95 \%)$, en el $57,29 \%$ de los casos el estadio de diagnóstico fue E0, El y EIIA y el 42,71\% desde EIIB hasta EIV.

Cuando se valoró la historia personal tumoral de las pacientes incluidas en el estudio, el 2,04\% casos registraban otros cánceres a lo largo de su vida, siendo los más frecuentes los cánceres asincrónicos y sincrónicos de mama. En cuanto a los antecedentes familiares con cáncer de mama en $1^{\circ}, 2^{\circ}$ y $1^{\circ}+2^{\circ}$ grado, fueron en encontrados 227 casos de las 285 fichas clínicas que presentaban esa información (ver figura 1).

Con la finalidad de analizar la tendencia de pre- 
sentación del cáncer de mama desde 2004/2010 en el ámbito público de la Ciudad de Córdoba, se efectuaron dos análisis de regresión, uno en relación a las modificaciones de la edad del diagnóstico en el periodo estudiado y la otra en relación al estadio del cáncer en el momento del diagnóstico. Se utilizó el modelo de regresión lineal para analizar la edad de las mujeres (variable respuesta) en función de los años de registro (variable regresora) a fin de establecer si en el periodo analizado se modifica la edad de diagnóstico de cáncer de mama en las mujeres que se asistieron en las instituciones involucradas. Así se encontró que por cada año de registro un incrementó en 0,39 en la edad de las mujeres que se registraron con diagnóstico de cáncer en dichas instituciones. Esto refleja que la edad de diagnóstico de cáncer de las mujeres registradas en estas instituciones se fue incrementando desde el 2004 al 2010.

En cuanto al tipo del estadio, se utilizó el modelo de regresión Poisson, en donde se efectuó un análisis de asociación entre el año del registro con el estadio de cáncer registrado en las historias clínicas de las pacientes. Dicho análisis no arrojó una relación entre las variables ( $p>0,05)$, lo que indicaría que no hay cambios en la tendencia del estadio en el período estudiado.

\section{DISCUSIÓN}

En los estudios de cáncer de mama, se observa que existe una marcada heterogeneidad entre los países y también entre regiones de un mismo país, respecto a la prevalencia de esta enfermedad y sus estadios; aludiendo a que esto puede deberse al desarrollo del sistema de salud económico-social de cada área estudiada. Teniendo en cuenta la edad del primer diagnóstico, el presente estudio refleja que el 8,74\% de las mujeres estudiadas eran menores a 39 años, mientras que la literatura mundial expresa valores que oscilan entre el $5 \%$ y $7 \% .{ }^{21} \mathrm{El}$ valor de este hallazgo se centra en que la franja de edad igual o menor a 39 años, presenta una epidemiología diferente al resto de las edades de las mujeres ${ }^{22}$. Los datos encontrados en mujeres menores de 39 años resultan relevantes al ser considerados como una población de riesgo, que debe ser tenida en cuenta para el diagnóstico precoz. Las estrategias para el diagnóstico de las mujeres más jóvenes son ecografías acompañadas de elastografías, lo cual incrementa el valor predictivo positivo, encontrando lesiones malignas sin masa palpable ${ }^{23}$. Por otra parte el exa- men mamográfico tiene una sensibilidad moderada y buena especificidad para la detección de cáncer, y los valores predictivos varían de acuerdo a la edad. La sensibilidad varía entre $57 \%$ y $83 \%$ entre los 18 años y los 50 y 59 años respectivamente. Entre los 40 y 49 años la sensibilidad es del $71 \%$. La especificidad por el contrario, es mayor: $88 \%$ a $96 \%$ entre 50 y 59 años y $71 \%$ a $84 \%$ en mujeres entre 40 a 49 años $^{24}$

En cuanto a la detección temprana Freitas y col (2015), refieren que las fallas en dicha detección o la falta de acceso a los tratamientos, explicarían una mayor mortalidad en los países en vías de desarroIlo. Al respecto más del $70 \%$ de las pacientes con cáncer de mama en países desarrollados son diagnosticadas en la etapa I o II, mientras que sólo el 20$50 \%$ de las que viven en países de medianos o bajos ingresos son diagnosticadas en etapas tempranas de la enfermedad.

Por otra parte el registro de la estadificación de la enfermedad es un aspecto relevante, dado que los autores refieren que la invasión de linfáticos axilares comprometidos, son un factor pronóstico importante para las pacientes con cáncer de mama, diferenciando el riesgo según se detecte en una etapa temprana o tardía ${ }^{23}$. En el presente estudio la proporción de mujeres mayores de 40 años detectadas en estadio temprano fue del $55,78 \%$ y un $44,22 \%$ fueron detectadas en un estadio tardío. Las pacientes con cáncer de mama en estadio ElIA quienes no tenían impacto axilar suman el 25,58\% del total de casos. El 15,18\% de los diagnósticos se presentaron en el estadio Ell B, el 22,05\% en el Elll y el 7,05\% en el EIV. Esta distribución debiera asumirse como un alerta desde la perspectiva de la salud pública, si se la compara con la informada por un estudio de Estados Unidos que presenta un 55\% de mujeres con E0 y El, un 35\% con Ell, un 6\% con Elll y menos del $5 \%$ con EIV 26 .

En cuanto a los antecedentes personales y familiares se considera que entre el $5-10 \%$ de todos los casos diagnosticados tienen un carácter heredofamiliar, de los cuales sólo 1\% se atribuye a síndromes genéticos y los restantes porcentajes a alteraciones de los genes BRCA 1 y 2, dada la probabilidad de padecer la enfermedad por determinados factores como edad, cargas genéticas y antecedentes patológicos familiares (APF) $(27,28)$. En el presente estudio, 41 del total de pacientes, sufrieron a lo largo de su vida, cáncer de diversas estirpes y en distintos órganos. Los más frecuentes fueron los cánceres de 
mama asincrónicos (aquellos ocurridos en tiempos diferentes $36,58 \%$ de los casos) y 24,39\% cánceres de mama sincrónicos (ocurridos al mismo tiempo); y con menor frecuencia cáncer de colon, tiroides, útero, ovarios.

En la última década se promueve las acciones para lograr un diagnóstico temprano, resaltando la importancia que tienen aquellas pacientes supuestas sanas con antecedentes familiares de riesgo, no solamente de mama, sino de otras variedades; en general no han sido tomados en cuenta en las historias clínicas, pudiendo alertar al especialista de una probable mutación genética en su paciente ${ }^{29}$. Al respecto, los factores a considerar en relación con la recomendación para las pruebas genéticas, incluyen la mutación conocida en la familia, o la paciente con familiar cercano con diagnóstico de cáncer de mama menor de 35 años con cáncer de ovario o de trompa de Falopio, o con antecedentes de cáncer de páncreas ${ }^{23}$. Sin embargo, y en relación a lo anteriormente explicitado, Chlebowski en su investigación explica que la mayoría de las neoplasias de mama son esporádicas (no hereditarias), se presume la predisposición hereditaria entre un 5\% - $10 \%$ ó 25\%, según el autor que se trate ${ }^{30}$.

La limitación del presente estudio radica en la focalización de la población analizada, ya que sólo se abordan los registros de los hospitales públicos de referencia en la provincia de Córdoba, Argentina. No obstante el interés fue analizar el perfil de los registros de mujeres con cáncer de mama durante una década, con la finalidad de aportar información que promuevan estrategias para la detección temprana del cáncer de mama, previniendo la muerte de cientos de mujeres, especialmente las que transitan edades económicamente activas.

\section{REFERENCIAS BIBLIOGRÁFICAS}

1. Ferlay J, Colombet $M$, Soerjomataram I, Mathers $C$, Parkin DM, Piñeros M, Znaor A, Bray F. Estimating the global cancer incidence and mortality in 2018: GLOBOCAN sources and methods. Int. J. Cancer: 144, 1941-1953 (2019).

2. Villarreal-Garza C, Lopez-Martinez EA, Muñoz-Lozano JF, Unger-Saldaña K. Locally advanced breast cancer in young women in Latin America. Ecancermedicalscience. 2019 Jan 22;13:894.

3. DeSantis C, Howlader N, Cronin KA, Jemal A. Breast cancer incidence rates in U.S. women are no longer declining. Cancer Epidemiol Bio-markers Prev. 2011.

4. Gajdos C, Tartter Paul I, Bleiweiss IJ, Bodian Carol, Brower Steven T. Stage 0 to stage III breast cancer in young women. J. Am. Coll. Surg. 2000; 190(5):523529.

5. Tazhibi Mahdi, Feizi Awat. Awareness Levels about Breast Cancer Risk Factors, Early Warning Signs, and Screening and Therapeutic Approaches among Iranian Adult Women: A large Population Based Study Using Latent Class Analysis. Biomed Res Int. 2014; 2014: 306352. Published online 2014 Sep 11. doi: $10.1155 / 2014 / 306352$

6. Vogel GV. Epidemiología, genética y evaluación del riesgo de cáncer de mama en mujeres pos menopáusicas. Revista del climaterio 2009 May/Jun. 12(70):12133.

7. Villarreal-Garza C, Lopez-Martinez EA, Muñoz-Lozano JF, Unger-Saldaña K. Locally advanced breast cancer in young women in Latin America. Ecancermedicalscience. 2019 Jan 22;13:894.

8. Viniegra M, Paolino M, Arrossi S. Cáncer de mama en Argentina: organización, cobertura y calidad de las acciones de prevención y control: Informe final julio 2010: diagnóstico de situación del Programa Nacional y Programas Provinciales. 1a ed. Buenos Aires: Organización Panamericana de la Salud - OPS, 2010.

9. Ministerio de Salud Información. Departamento de Estadísticas e Información en Salud, DEIS. (2011).

10. Armando E. Giuliano, James L. Connolly, Stephen B. Edge, Elizabeth A. Mittendorf, Hope S. Rugo, Lawrence J. Solin, Donald L. Weaver, David J. Winchester, Gabriel N. Hortobagyi. Breast Cancer-Major Changes in the American Joint Committee on Cancer Eighth Edition Cancer Staging. CACANCER J CLIN 2017;67:290-303.

11. Ferlay J, Shin HR, Bray F, Forman D, Mathers C, Parkin D. M. GLOBOCAN 2008, Cancer Incidence and Mortality Worldwide: IARC Cancer Base No. 10 [Internet]: Lyon, France: International Agency for Research on Cancer. Disponible en: http://www.iarc.fr/en/media-centre/iarcnews/2010/globocan2008.php

12. Karimi Asrin, Delpisheh Ali, Sayehmiri Kourosh, et al. Predictive Factors of Survival Time of Breast Cancer in Kurdistan Province of Iran between 2006-2014: A Cox Regression Approach. Asian Pac J Cancer prev.2014;15(19):8483-8488. 
13. Hortobágyi GN, de la Garza Salaza J, Pritchard K, Amadori D, et al. The global breast cancer burden: variations in epidemiology and survival. Clin Breast Cancer. 2005; 6(5): 391-401.

14. Miranda M Fidler, Sumit Gupta, Isabelle Soerjomataram, Jacques Ferlay, Eva Steliarova-Foucher, Freddie Bray. Cancer incidence and mortality among young adults aged 20-39 years worldwide in 2012: a population-based study. Lancet Oncol 2017; 18: 1579-89.

15. Boyle P. Triple-negative breast cancer: epidemiological considerations and recommendations. Ann Oncol 2012; 23(suppl 6): vi7-vi12.

16. 16. Newman L. Biology of breast cancer in young human. CDC Committee on brast cancer in young human. Michigan; University of Michigan. 2013. Disponible en: http://www.cdc.gov/cancer/breast/pdf/Newman_Biology_of_Breast_Cancer_in_Young_Women. pdf

17. Edge SB, Compton CC. The American Joint Committee on Cancer: the 7th edition of the AJCC cancer staging manual and the future of TNM. Ann Surg Oncol. 2010;17(6):1471-4. Disponible en: http://www.ncbi. nlm.nih.gov/pubmed/20180029

18. Assi HA, Khoury KE, Dbouk H, Khalil LE, Mouhieddine TH, El Saghir NS. Epidemiology and prognosis of breast cancer in young women. J Thorac Dis. 2013;5(Suppl 1):S2-S8.

19. Hayat M J, Howlader N, Reichman ME, Edwards BK. Cancer Statistics, Trends, and Multiple Primary Cancer Analyses from the Surveillance, Epidemiology, and End Results (SEER) Program. Oncologist. 2007; 12(1): 20-37.

20. Clavel-Chapelon F, Gerber M. Reproductive factors and breast cancer risk. Do they differ according to age at diagnosis? Breast Cancer Res Treat. 2002;72(2):10715.

21. Escrich E, Moral R, Solanas M. Olive oil, an essential component of the Mediterranean diet, and breast cancer. Public Health Nutrition. 2011;14(12A):2323-32.

22. Collaborative Group on Hormonal Factors in Breast Cancer. Breast cancer and breastfeeding: collaborative reanalysis of individual data from 47 epidemiological studies in 30 countries, including 50302 women with breast cancer and 96973 women without the disease. Lancet. 2002; 360(9328):187-95.

23. Goldhirsch A, Winer EP, Coates AS, Gelber RD, Piccart-Gebhart M, Thürlimann B, Senn HJ, Panel members. Personalizing the treatment of women with early breast cancer: highlights of the St Gallen International Expert Consensus on the Primary Therapy of Early Breast Cancer 2013. Ann Oncol. 2013; 24(9):22062223.

24. Celso Pinto Nazario A, Facina G, Filassi JR. Breast cancer: news in diagnosis and treatment. Rev Asso C Med Bras. 2015. 61(6): 543-552.

25. Freitas AGQ, Weller M. Patient delays and system delays in breast cancer treatment in developed and developing countries. Ciência \& Saúde Coletiva. 2015; 20 (10): 3177-3189.

26. Yip $\mathrm{CH}$, Smith RA, Anderson BO, Miller AB, Thomas DB, Ang ES, Caffarella RS, Corbex M, Kreps GL, McTiernan A. Breast Health Global Initiative Early Detection Panel. Guideline implementation for breast healthcare in low- and middle-income countries: treatment resource allocation. Cancer. 2008; 113 (8 Suppl): 2269-2281.

27. 27. Ramos Aguila YC, Marimón Torres ER, Crespo González C, Junco Sena B, Valiente Morejón W. Cáncer de mama, su caracterización epidemiológica. Rev. Ciencias Médicas. 2015; 19 (4): 619-629.

28. Núñez Copo AC, Frómeta Montoya Cl, Rubio González T. Factores ambientales y genéticos asociados al cáncer de mama en féminas del área de salud 28 de Septiembre. MEDISAN [Internet]. 2011 Feb[citado20 julio 2016]; 15(2):[aprox 7p.]. Disponible en: http:// bvs.sld.cu/revistas/san/vol_15_2_11/san03211.htm

29. Petit JY, Botterim E, Lohsiriwat V, et al. Locoregional recurrence risk after lipofilling in breast cancer patients. Ann Oncol 2012; 23: 582-588.

30. Chlebowski RT. Nutrition and physical activity influence on breast cancer incidence and recurrence. Breast 2013; 22(Supp 2); S30-7. 